\title{
Effect of Acupressure by Sea-Bands on Nausea and Vomiting of Pregnancy
}

\author{
Nancy M. Steele, RNC, MSN, WHNP, Joyce French, RN, PhD, \\ Janet Gatherer-Boyles, RN, MM, Sandra Newman, RN, MSN, WHNP, \\ Suzanne Leclaire, RN, MHS
}

Objective: To determine the effect of continuous acupressure at $P_{6}$ applied by Sea-Bands with acupressure buttons on the frequency and severity of nausea and vomiting of pregnancy during the 1st trimester.

Design: A two-group, quasi-experimental, posttest-only and posttest-repeated measure.

Selting: Seventeen medical clinics or offices in southern Michigan.

Participants: Convenience sample of Englishspeaking, healthy pregnant women in their 1 st trimester, who had at least one episode of nausea, vomiting, or both before their prenatal clinic/office visit where they were recruited. After being accepted for the study, the women were randomly assigned to treatment or placebo groups.

Intervention: Treatment group 1 applied SeaBands with acupressure buttons to both wrists for 4 days and removed the Sea-Bands for 3 subsequent days. Placebo group 2 applied the Sea-Bands without acupressure buttons to both wrists on the same time schedule as group 1.

Main Outcome Measure: Self-report daily diaries of the number of times per day that participants experienced nausea, the severity of nausea, the number of vomiting episodes per day, and the severity of vomiting.

Results: Mann-Whitney U procedures revealed that the treatment group had significantly less frequency and severity of nausea and vomiting of pregnancy while wearing the Sea-Bands than did the placebo group. The treatment group also had significantly less frequency and severity of nausea and vomiting of pregnancy while wearing the SeaBands than when not wearing the Sea-Bands.

Conclusions: Sea-Bands with acupressure buttons are a noninvasive, inexpensive, safe, and effective treatment for the nausea and vomiting of pregnancy. JOGNN, 30, 61-70; 2001.

Keywords: Acupressure-Alternative therapies-Morning sickness-Nausea-PregnancySea-Bands-Vomiting

Accepted: June 2000

Nausea with or without vomiting during pregnancy is experienced by $50-70 \%$ of pregnant women and is a universal phenomenon that occurs most frequently between weeks 5 and 12 of gestation (Murphy, 1998; O'Brien \& Zhou, 1995; Pillitteri, 1995). Although the condition is usually self-limited by the end of the 1st trimester, the symptomatic period may lead to nutritional deficits, dehydration, weakness, weight loss, and electrolyte imbalance (Murphy, 1998; Stainton \& Neff, 1994). Nearly one third of women with nausea and/or vomiting of pregnancy (NVP) report considerable distress and disruption in family, emotional, or social functioning (O'Brien \& Naber, 1992). In affected women who are employed, nearly $50 \%$ believe that their efficiency at work is reduced, and $25 \%$ require time off (Vellacott, Cooke, \& James, 1988).

The etiology of NVP is not clearly understood. Findings from epidemiologic studies that evaluated relationships between NVP and a variety of 
physiologic, psychologic, psychosocial, and demographic variables have been equivocal and even contradictory. Some investigators have reported a positive association between symptoms and maternal factors (personality factors, age, parity, education level, weight, smoking habits) and fetal factors (gender, birth weight), whereas others failed to support these associations (Depue, Bernstein, Ross, Judd, \& Henderson, 1987; Hsu \& Witter, 1993; Janfelt-Samsioe, Samsioe, \& Velinder, 1983; Klebanoff, Koslowe, Kaslow, \& Rhodes, 1985; O’Brien, 1991; O'Brien \& Zhou, 1995; Tierson, Olsen, \& Hook, 1986).

Because there is no definitive maternal or fetal cause, few effective treatment options are available to women experiencing NVP. Since the 1940 s, vitamin $\mathrm{B}_{6}$ (pyridoxine) has been included in the formulation of drugs for the prevention of NVP (Weinstein, Wohl, Mitchell, \& Susendal, 1944; Willis, Winn, Morris, Newsom, \& Massey, 1942). Many health care providers continue to re-

\section{4 - \\ Where is a great need for a safe, effective, and side-effect-free intervention for the nausea and vomiting of pregnancy.}

commend vitamin $B_{6}$ for control of NVP, yet studies provide only minimal support for the relationship between vitamin $\mathrm{B}_{6}$ and prevalence or degree of NVP (Sahakian, Rouse, Sipes, Rose, \& Niebyl, 1991; Vutyavanich, Wongtrangan, \& Ruangsri, 1995). Before 1983, a medication containing a combination of vitamin $B_{6}$ and doxylamine succinate provided an effective antiemetic; however, evidence that the vitamin $B_{6}$ component contributed to its effectiveness was never supported (Dilorio, 1988). The medication was withdrawn from the market in 1983 after several lawsuits alleging birth defects related to its use (Ornstein, Einarson, \& Koren, 1995).

Although some pharmacologic antiemetics are available, many pregnant women are reluctant to take them for fear of possible teratogenic effects on the fetus during this critical embryogenic period (Murphy, 1998). The Food and Drug Administration lists most such medications as Category C. This category indicates that there is minimal information about the drugs concerning safety or risk in humans.

Acupressure is considered one of the nonpharmacologic and noninvasive treatment modalities for NVP. Others are alterations of eating and drinking patterns, vitamins, herbals, and homeopathic preparations (Mur- phy, 1998). Few women report complete relief from these modalities, and their potential risks and adverse effects are unknown (O'Brien \& Naber, 1992). Clearly, with no dependable nonpharmacologic treatment available, there is a great need for an intervention that is safe, effective, and free of side effects. In recent years, acupressure has been looked on as a treatment modality possibly meeting these criteria. It is a noninvasive, inexpensive, and safe treatment that may be a successful self-help option for pregnant women experiencing NVP. All of these modalities need to be studied more thoroughly.

\section{Theoretical Framework}

There is no known physiologic basis for acupressure. According to Beal (1992a, 1992b), acupressure is based on ancient Chinese traditions reflecting the philosophies of Taoism and Confucianism. These philosophies describe health as a state of relative balance within the person and in the context of his or her environmental surroundings. The basic life force of ch'i empowers human life. Ch'i flows through the body in meridians (pathways) that are accessible to acupressure and acupuncture treatments that change the flow imbalances. According to Chinese medicine, acupressure for NVP involves bilateral wrist pressure that rebalances energy so that the negative energy in the body is first redirected away from the heart using the right $P_{6}$ point, whereas more positive energy comes into the body through the left $P_{6}$ point. This process restores the balance of the life forces and controls nausea (Stainton \& Neff, 1994). Acupressure for NVP involves constant pressure of the pericardial median at the Neiguan $\left(\mathrm{P}_{6}\right)$ point, located three finger breadths from the wrist crease on the volar surface of the arm between the palmaris longus and flexor carpi radialis.

Many meridian-oriented treatment approaches for acupressure require a high level of knowledge and experience to apply properly (Beal, 1992a). One simple alternative acupressure intervention that requires minimal knowledge and experience is the Sea-Band wristband (Sea-Band, U.K., Ltd., Leicester, England). SeaBands are 1-inch-wide elasticized cloth wristbands with a convex plastic button that is worn against the skin. They are packaged with insert instructions illustrating exactly where to place them on the wrists to exert even pressure on the $P_{6}$ point (see Figure 1).

\section{Literature Review}

The dearth of research supporting or refuting the efficacy of all remedies has left a therapeutic gap in the treatment of NVP. This problem, along with the current 


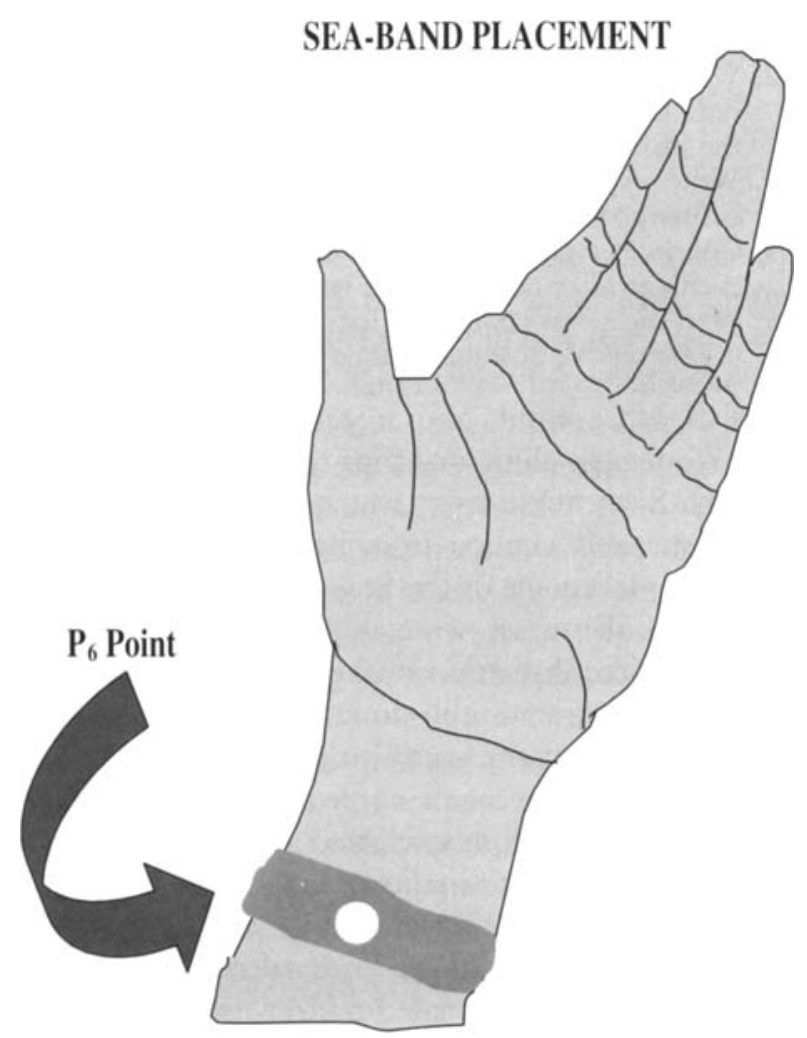

FIGURE 1

Placement of Sea-Bands with acupressure button at the $P_{6}$ point located three fingers below the wrist crease.

and increasing interest in complementary medicine, led researchers to investigate the use of acupressure. Six clinical trials studying the efficacy of acupressure at the Neiguan point $\left(\mathrm{P}_{6}\right)$ as an antiemetic for NVP were identified through a literature review. Only one study reported no effectiveness (O'Brien, Relyea, \& Taerum, 1996), whereas the other five demonstrated some support for the antiemetic action of $P_{6}$ acupressure (Bayreuther, Lewith, \& Pickering, 1994; Belluomini, Litt, Lee, \& Katz, 1994; De Aloysio \& Penacchioni, 1992; Dundee, Sourial, Ghaly, \& Bell, 1988; Hyde, 1989).

One of the most recent studies (O'Brien et al., 1996) demonstrated no apparent medical benefit from the use of $P_{6}$ acupressure. This study used Sea-Bands on both wrists and performed experimental and placebo tests on 161 pregnant women in their 1st trimester. All participants were assigned to one of three groups (i.e., $P_{6}$ acupressure, placebo acupressure bands inappropriately placed, or control) on the basis of a process of blocked randomization. One hundred forty-nine pregnant women completed the protocol. Irrespective of group assignment, the women reported decreases in nausea and vomiting or retching. Thus, there was no differential treatment effect in reducing or relieving the symptoms of nausea with or without vomiting and retching during pregnancy as a result of acupressure.

Three of the studies found that during the 1st trimester, acupressure at the $P_{6}$ point may reduce nausea but not vomiting (Bayreuther et al., 1994; Belluomini et al., 1994; Hyde, 1989). In the other two studies that demonstrated support, the collection methods did not permit separate analysis for nausea and vomiting (DeAloysio \& Penacchioni, 1992; Dundee et al., 1988). In Dundee et al.'s (1988) study, the treatment group reported $8.5 \%$ reduction in nausea and vomiting. However, the gestational age was significantly greater in this group. Because the study did not use a crossover design, it is unknown if gestational age in itself may have accounted for the lower reporting of nausea and vomiting (Dundee et al., 1988). Also, there was no description in Dundee's report of how acupressure was applied or any explanation for the significantly lower rates of return in the treatment group. Hyde (1989) used a crossover design, and 12 of the 16 participants reported a reduction in nausea and vomiting in pregnancy. In Hyde's study, the sample was small and there was no control group for study of a placebo effect. The sample in the study by Bayreuther et al. (1994) also was small, with a high dropout rate. Although the studies of Hyde and Bayreuther support the use of acupressure, one must be cautious when the sample is so small.

Review of the literature reveals an incomplete understanding of NVP, lack of effective treatment modalities, and inconclusive support for the use of acupressure. This leaves nurses without an effective intervention based on sound research. Clearly, the review of the literature confirms the need for nurses to study the efficacy of acupressure, which is cost-effective, free of side effects, and noninvasive. It has gained rather rapid

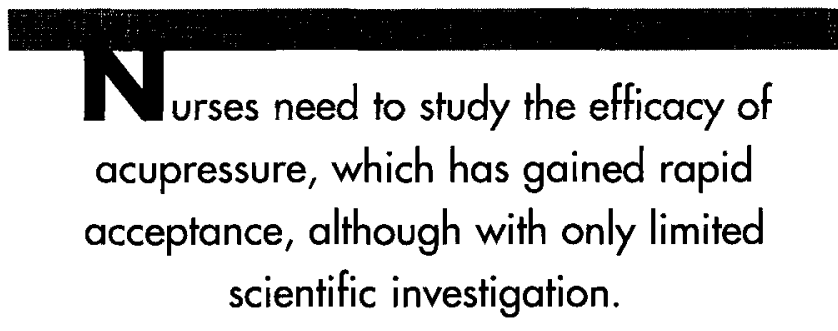

acceptance with only limited scientific investigation. For nurses to confidently offer Sea-Bands as a remedy for NVP, the effectiveness of the intervention requires further evaluation. The purpose of this investigation was to determine if continuous acupressure at $\mathrm{P}_{6}$ applied by Sea-Bands decreased the frequency and severity of nausea and vomiting in pregnancy during 
the 1st trimester. The current study tested the following hypotheses:

1. The frequency and severity of nausea will be less among pregnant women wearing acupressure SeaBands than among women wearing placebo Sea-Bands.

2. The frequency and severity of vomiting will be less among pregnant women wearing acupressure SeaBands than among women wearing placebo Sea-Bands.

3. The frequency and severity of nausea will be less when pregnant women are wearing acupressure Sea-Bands than when they are not wearing the bands.

4. The frequency and severity of vomiting will be less when pregnant women are wearing acupressure SeaBands than when they are not wearing the bands.

\section{Design and Method}

A two-group, quasi-experimental, posttest-only and posttest-repeated measure design (Campbell \& Stanley, 1963) was used to address the research question, Does continuous acupressure at $\mathrm{P}_{6}$ applied by Sea-Bands decrease the frequency and severity of nausea and vomiting of pregnancy during the 1 st trimester? To address this question, a convenience sample of pregnant women in their 1st trimester was recruited on a voluntary basis in 17 obstetric/gynecology offices and clinics in southern Michigan. Criteria for participation were (a) selfreport of one or more episodes of pregnancy-related nausea and/or vomiting, (b) less than 13 weeks pregnant, and (c) able to read and speak English. The design also provided for the participants to serve as their own control (when they removed the Sea-Bands) for the last 3 days of the study period.

After the university subject review board approved the study design and instruments, participants were recruited through nurses at the participating offices and clinics. All women who met the inclusion criteria were asked to participate. Assignment of participants to group 1 (treatment, Sea-Band with acupressure button) or group 2 (placebo, Sea-Band without acupressure button) occurred after the pregnant women agreed to participate in the study and signed the consent form. Each office or clinic was given a box containing an equal number of treatment and placebo packets. To prevent identification as to whether treatment or placebo, the unlabeled sealed packets were shuffled before delivery to the offices.

An explanation of the study, a cover sheet, a consent form, and a demographic questionnaire were stapled to the outside of the sealed packet. Inside the sealed packet were written instructions, seven daily logs, and SeaBands with or without acupressure buttons. Written instructions to the participants included the following:

1. Place one wristband on each wrist three finger breadths from the wrist crease. (If the wristband had an acupressure button, the nurse also instructed the partici- pant to place the button as illustrated and directed on the Sea-Band package.)

2. Wear the wristbands continuously for 4 days. (The removal date and time [ 96 hours later] were written on the instruction sheet for participants.)

3. Write responses on the dated daily log each evening just before going to bed.

4. Continue writing responses on the daily logs for 3 evenings after removing the wristbands.

5. Mail the seven completed logs to the investigators in a preaddressed, postage-paid envelope.

Investigators conducted training sessions with the nurses from each clinic or office that agreed to assist in the study. Each nurse was given a script for instructing participants and explicit information and demonstration in the placement of the Sea-Bands. After the training session, all nurses were required to give a return proficiency demonstration in the study methods.

All trained nurses in the clinics or offices read a written explanation of the study to potential participants. When a pregnant woman agreed to take part in the study, the nurse randomly selected a sealed packet from the study box. Before opening the sealed packet, the participant signed a consent form and completed a demographic questionnaire and a cover sheet. The nurse then opened the sealed packet and removed the contents. The participants were not informed of which type of Sea-Band they had been given. Both groups 1 and 2 received detailed written and oral instructions for the logs and Sea-Bands. The participants from both groups applied a Sea-Band on each of their wrists with the assistance of the nurses. The nurses made certain that participants used their own fingers to measure three finger breadths from the creases in their wrists. Group 1 participants also were instructed to place the pressure button between flexor tendons of the medial aspect of both forearms. These instructions were consistent with those provided by the Sea-Band distributor. This day was considered the 1 st of the 7 days of the study for the participant.

Both groups were instructed to wear the wristbands continuously (remove only when bathing), while concurrently maintaining the daily log for 4 days. At the end of the 96th hour, both groups were instructed to remove the wristbands but to continue to maintain the daily diaries for an additional 72 hours. The control period was limited to 72 hours to reduce nonadherence in symptomatic women who might be tempted to put the wristbands back on if they had relief while wearing the SeaBands. On completion of the 72nd hour, they mailed the daily logs back to the investigators. When the investigators received a placebo-coded log, the participant was telephoned and offered a free pair of Sea-Bands with a pressure button. This gesture was provided because the investigators felt that each participant, whether 
in the treatment or placebo group, should have an opportunity to use the Sea-Bands with acupressure buttons.

\section{Instruments}

A self-report demographic questionnaire was used to obtain background information. The questionnaire was designed specifically for this study to include contextual factors, which according to the literature reviewed, could affect the results. The factors included were age, gestational age, race, education, marital status, gravida, first NVP episode, nausea proneness, vitamin use, importance of significant other, and type of medical insurance. A cover sheet was used to collect identifying information for each participant. It was used only for sending follow-up reminder postcards if logs were not received and for sending those in the placebo group free Sea-Bands after the study was completed.

Each participant recorded her perception of the frequency and severity of nausea and vomiting for 7 days in a daily log. The daily log was a modified and abbreviated version of the Rhodes Index of Nausea and Vomiting (Rhodes, Watson, \& Johnson, 1984), which had been adapted by Brown, North, Marvel, and Fons (1992) for their study of acupressure wristbands to relieve nausea and vomiting in hospice patients. The Rhodes Index of Nausea and Vomiting has been used extensively in research and has an established validity with populations of pregnant women (O'Brien, 1991; Stainton \& Neff, 1994). The instrument has an estimated reliability of at least 0.83 and a correlation of .37 between its construct and concurrent validity (Rhodes et al., 1984). The version of the instrument used in the current study was that used in the Brown et al. (1992) study, with two changes. In the Brown et al. version, the third question asked participants how long they were nauseated. The version used in this study asked women how many times they were nauseated. In this study, a fifth question about the wearing of bands also was added.

Therefore, the four closed-ended questions used to measure participants' perception of their frequency and severity of nausea and vomiting were (a) How many times today did you feel nauseated or queasy? (b) How bad was your nausea or queasiness today? (c) How many times did your throw up (vomit) or have dry heaves today? and (d) How bad was your vomiting/dry heaves today? Responses were recorded on a 5 -point Likert-type scale for frequency $10=$ none, $1=$ one to two times, $2=$ three to four times, $3=$ five to six times, and $4=$ seven or more times $)$ and for severity $(0=$ none, $1=$ mild, $2=$ moderate, $3=$ severe , and $4=$ borrible $)$. $\mathrm{A}$ fifth question was used to measure the participants' perception of whether the Sea-Bands helped to reduce
NVP: 1 = did not help, 2 = helped a little, $3=$ belped $a$ lot, 4 = did not wear bands today. This fifth question also assisted the investigators in confirming whether the participants actually had taken the Sea-Bands off for the last 3 days of the 7-day study.

Because vitamin $B_{6}$ and prenatal vitamins are routinely recommended by the participating clinics and offices, the logs also included questions as to whether the women took either of these supplements. An additional six questions about the number of large or small meals consumed and the amount and type of fluid intake also were included in the daily logs.

\section{Results}

One hundred thirty-eight pregnant women in 17 different prenatal clinics or offices in southern Michigan accepted the invitation to participate in this study. Of these, 110 participants completed the data collection and mailed their results to the investigators. The remaining 28 were not included because of insufficient data provided. Of the 28,17 were in the treatment group and 11 were in the placebo group. No other information was available on the women who agreed to participate but did not complete the study.

Sixty-eight of the completed logs were from women in group 1 (treatment, Sea-Bands with acupressure buttons) and 42 were from women in group 2 (placebo, SeaBands with no acupressure buttons). Mann-Whitney $U$, chi-square, and Fisher's exact tests revealed that the groups were not significantly different in background characteristics (see Table 1).

This research was guided by the following question: Does continuous acupressure at $\mathrm{P}_{6}$, applied by SeaBands, decrease the frequency and severity of NVP during the 1st trimester? The first two hypotheses were tested to address the question. Mann-Whitney U procedures were used to determine if there were measurable differences between group 1 and group 2 in the frequency and severity of NVP during the 4-day treatment period. All four research hypotheses were supported (see Table 2).

The nonparametric procedure, Wilcoxin's matched pairs, signed-rank test, was used to test for the differences between Days 1-4 and Days 5-7, when the women served as their own experimental controls. The last two research hypotheses were supported (see Table 3 ).

Although no hypotheses were identified comparing the groups' results for Days 5-7, the results are included here for further discussion. The medians for all four measures were higher for Days 5-7 than for Days 1-4. Differences in the frequency and severity of nausea and vomiting were less between group 1 and group 2 during Days 5-7 than during the treatment period Days 1-4. 
TABLE 1

Sample Description: Background Variables of Participants $(\mathrm{N}=110)$

\begin{tabular}{|lllc|}
\hline Variable & \multicolumn{1}{c|}{ Group 1 (n= 68) } & \multicolumn{1}{c|}{ Group 2 $(\mathrm{n}=42)$} & Statistic \\
Age $(M)$ & 27 years & 28 years & $1,238.5$ \\
Gestation $(M)$ & 57 days & 60 days & 1,032 \\
Race & $60 \%$ white & $61 \%$ white & .290 \\
Education & $87 \%$ high school graduate & $94 \%$ high school graduate & \\
Marital status & 63\% married & $66 \%$ married & .657 \\
Gravida & $45 \%$ primipara & $48 \%$ primipara & .084 \\
Nausea prone & $43 \%$ easily & $47 \%$ easily & .196 \\
Insured & $69 \%$ private & $74 \%$ private & .859 \\
\hline
\end{tabular}

The tests for probability for Days 5-7, however, showed significant differences between treatment and placebo groups only in frequency of nausea and severity of vomiting but not in severity of nausea or frequency of vomiting (see Table 4).

Table 5 shows the results of the Wilcoxin's matched pairs, signed-rank tests for group 2. This group showed significantly less frequency and severity of vomiting during the time they wore the placebo Sea-Bands (Days 14) than after they removed them (Days 5-7). There were no differences in either frequency or severity of nausea.

Because many of the women in both group $1(40 \%)$ and group $2(37 \%)$ were taking vitamin $\mathrm{B}_{6}$ during the 7 study days, further investigation into the possibility of a treatment effect of vitamin $B_{6}$ seemed warranted. To separate any effects exerted on NVP by vitamin $B_{6}$, the two groups were divided again. Group 1 was divided into nontakers and takers of vitamin $B_{6}$, as was group 2 . Medians for frequency and severity of NVP were calculated for each of the four groups and tested for differences with the Mann-Whitney U. Within the treatment group, during treatment Days 1-4, the takers of vitamin $B_{6}$ had significantly less frequency and severity of NVP than did the nontakers of vitamin $B_{6}$. Within the placebo group, during Days 1-4, the takers also showed significantly less frequency and severity of NVP than did the nontakers (see Tables 6 and 7). There were

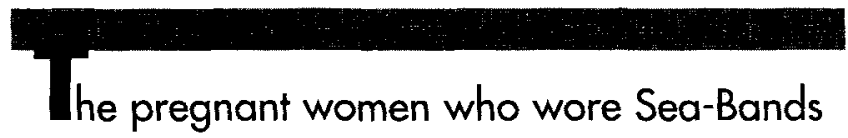
with acupressure buttons had less frequency and severity of nausea and vomiting of pregnancy than did the women who wore Sea-Bands without acupressure buttons.

66 JOGNN
TABLE 2

Mann-Whitney $U$ Comparison of Independent Groups: Group 1 and Group 2 During Days 1-4 (wearing Sea-Bands)

\begin{tabular}{|llcc|}
\hline Variable & n & Mean Rank & $U$ \\
Nausea frequency & & & \\
Group 1 & 68 & 40.30 & $499 * *$ \\
Group 2 & 42 & 72.82 & \\
Nausea severity & & & \\
Group 1 & 68 & 40.13 & $488.5^{* *}$ \\
Group 2 & 42 & 73.09 & \\
Vomiting frequency & & & \\
Group 1 & 68 & 41.51 & $576.5^{* *}$ \\
Group 2 & 42 & 70.94 & \\
Vomiting severity & & & \\
Group 1 & 68 & 39.28 & $434.0^{* *}$ \\
Group 2 & 42 & 73.65 & \\
* * .0005, one-tailed. & & & \\
\hline
\end{tabular}

no significant differences between the takers and the nontakers of vitamin $B_{6}$ within either group 1 or group 2 during Days 5-7. These were days neither group 1 nor group 2 wore the Sea-Bands (see Tables 8 and 9).

\section{Discussion}

The 68 pregnant women who wore Sea-Bands with acupressure buttons (treatment group) had significantly less frequency and severity of NVP while wearing the Sea-Bands than did the 42 pregnant women who wore Sea-Bands without acupressure buttons (placebo group). Thus, the first two research hypotheses were accepted.

The treatment group also had significantly less frequency and severity of NVP while wearing the Sea-

66. 
TABLE 3

Results of Wilcoxin's Matched Pairs, Signed-Rank Test Within Group 1: Differences Between Experimental Period Days 1-4 and Control Period Days 5-7

\begin{tabular}{|ccccc|}
\hline & \multicolumn{2}{c}{ Nausea } & \multicolumn{2}{c|}{ Vomiting } \\
Test Statistic & Frequency & Severity & Frequency & Severity \\
$Z$ & $-5.285^{* *}$ & $-5.394^{* *}$ & $-4.736^{* *}$ & $-5.287^{* *}$ \\
$* * p<.0005$, one-tailed. & & & & \\
\hline
\end{tabular}

TABLE 4

Mann-Whitney $U$ Comparison of Independent Groups (1 = treatment; 2 = placebo) During Days 5-7 (not wearing Sea-Bands)

\begin{tabular}{|lccc|}
\hline & nean & $\begin{array}{c}\text { Mank } \\
\text { Raverage frequency of nausea }\end{array}$ & $U$ \\
$\quad$ & & & $956.5^{*}$ \\
Group 1 & 68 & 47.18 & \\
Group 2 & 42 & 58.47 & \\
Average severity of nausea & & & $1,100.0$ \\
$\quad$ Group 1 & 68 & 49.46 & \\
$\quad$ Group 2 & 42 & 54.79 & \\
Average frequency of vomiting & & & $1,161.5$ \\
$\quad$ Group 1 & 68 & 50.44 & \\
Group 2 & 42 & 53.22 & \\
Average severity of vomiting & & & $922.0^{*}$ \\
$\quad$ Group 1 & 68 & 46.63 & \\
Group 2 & 42 & 58.24 & \\
* $p<.05$, one-tailed. & & & \\
\hline
\end{tabular}

Bands with the acupressure button (Days 1-4) than they did after removal (Days 5-7). Thus, the last two hypotheses were accepted.

The treatment group had significantly less frequency of nausea and less severity of vomiting during Days 5-7 than did the placebo group. Although the medians for all four measures were less for the treatment group than the placebo group, there was no statistical difference in severity of nausea or frequency of vomiting between the groups. This could be a residual effect of the Sea-Bands for 24 hours after the bands are removed. To investigate this, Mann-Whitney U tests were used on Days 6 and 7, eliminating Day 5. Nearly identical results were found, ruling out any residual effect of Sea-Band treatment in this study.

The placebo group, when using themselves as controls (Days 5-7), showed significantly less frequency and severity of vomiting during Days 1-4 when they wore the placebo Sea-Bands than they did after remov- ing the Sea-Bands. However, there were no significant differences in either frequency or severity of nausea within this group between the time they wore the placebo Sea-Bands and when they did not. This may reflect a lack of clarity in reporting because one would expect a feeling of nausea to precede actual vomiting. Thus, all four tests indicated that acupressure through Sea-Bands is an effective remedy for NVP.

The test for the influence of vitamin $B_{6}$ further confirmed the effect of the Sea-Bands. The takers of vitamin $B_{6}$ in the treatment group had significantly less frequency and severity of NVP during the first 4 days than did the nontakers. Whereas Sea-Band treatment had lowered NVP overall, this finding suggests that there may be a positive, synergistic effect of the dual treatment. During Days 5-7, after Sea-Band removal, there were no significant differences between the takers and nontakers of vitamin $B_{6}$ among participants in the treatment group.

The effect of vitamin $B_{6}$ was similar in both the treatment and placebo groups. Vitamin $B_{6}$ was effective while participants wore Sea-Bands but not effective when they did not wear them. In this case, one has to question if a placebo effect occurred with the group wearing Sea-Bands without acupressure buttons. Is it possible to have a synergistic effect with a placebo Sea-Band? When both groups removed the Sea-Bands, the vitamin $\mathrm{B}_{6}$ made no difference. Therefore, vitamin $\mathrm{B}_{6}$, by itself, cannot be considered to be an effective treatment.

\section{Limitations}

A limitation associated with this study was the use of ordinal rather than interval data, which reduced the power of the findings. Thus, the recommendation for further research is to use actual numbers rather than ranges. A power analysis based on Cohen's (1992) formula determined that the sample size necessary to achieve an anticipated medium effect size with a power of 0.80 and an alpha of .05 was 170 ( 85 in each group). The final sample size in this study was 110 , with 68 in group 1 and 42 in group 2 . A limitation with regard to the sample size is that if a larger sample with 85 in 
TABLE 5

Results of Wilcoxin's Matched Pairs, Signed-Rank Test Among Group 2 (placebo): Differences Between Experimental Period Days 1-4 and Control Period Days 5-7

\begin{tabular}{|c|c|c|c|c|}
\hline \multirow[b]{2}{*}{ Test Statistic } & \multicolumn{2}{|c|}{ Nausea } & \multicolumn{2}{|c|}{ Vomiting } \\
\hline & Frequency & Severity & Frequency & Severity \\
\hline Z & -.820 & -1.555 & $-1.849^{*}$ & $-2.383^{*}$ \\
\hline
\end{tabular}

TABLE 6

Mann-Whitney $U$ Comparison of Independent Samples: Nontakers of Vitamin $B_{6}(\mathbf{n}=$ 35) and Takers of Vitamin $B_{6}(\mathrm{n}=29)$ Within Group 1 Participants (treatment), Days 1-4

\begin{tabular}{|lcc|}
\hline & Mean Rank & $U$ \\
Average frequency of nausea & & $38.0^{*}$ \\
Nontakers of vitamin $B_{6}$ & 37.34 & \\
Takers of vitamin $B_{6}$ & 26.66 & \\
Average severity of nausea & & $363.0^{*}$ \\
$\quad$ Nontakers of vitamin $B_{6}$ & 36.63 & \\
Takers of vitamin $B_{6}$ & 27.52 & \\
Average frequency of vomiting & & $388.0^{*}$ \\
$\quad$ Nontakers of vitamin $B_{6}$ & 35.91 & \\
Takers of vitamin $B_{6}$ & 28.38 & $395.5^{*}$ \\
Average frequency of vomiting & & \\
Nontakers of vitamin $B_{6}$ & 35.70 & \\
Takers of vitamin $B_{6}$ & 28.64 & \\
& & \\
" $p<.05$, one-tailed. & & \\
\hline
\end{tabular}

each group had been used, there would have been a reduced risk of possibly committing a Type I error. Another limitation was that the frequency and severity of NVP was not determined before participants enrolled in the study. They were required to have had at least one episode but were not asked to provide additional data. A pretest would have provided one more comparison and strengthened the study. It would have been interesting to determine the effect of the SeaBands on subsets of participants who experienced moderate or greater nausea and vomiting versus those who had mild or less nausea and vomiting, but this was not done in this study. The validity of self-reporting is always in question.

\section{Implications for Nursing}

Today, more than ever, health care reform calls on nursing to provide cost-effective care. Nurses are respon-
TABLE 7

Mann-Whitney $U$ Comparison of Independent Samples: Nontakers of Vitamin $B_{6}(\mathrm{n}=$ 22) and Takers of Vitamin $B_{6}(\mathbf{n}=19)$ Within Group 2 (placebo), Days 1-4

\begin{tabular}{|c|c|c|}
\hline & Mean Rank & $U$ \\
\hline Average frequency of nausea & & $120.5^{*}$ \\
\hline Nontakers of vitamin $B_{6}$ & 16.98 & \\
\hline Takers of vitamin $B_{6}$ & 25.66 & \\
\hline Average severity of nausea & & $101.5^{*}$ \\
\hline Nontakers of vitamin $B_{6}$ & 16.11 & \\
\hline Takers of vitamin $B_{6}$ & 26.66 & \\
\hline Average frequency of vomiting & & $148.5^{*}$ \\
\hline Nontakers of vitamin $B_{6}$ & 18.25 & \\
\hline Takers of vitamin $B_{6}$ & 24.18 & \\
\hline Average severity of vomiting & & $110.5^{*}$ \\
\hline Nontakers of vitamin $B_{f}$ & 16.26 & \\
\hline $\begin{array}{l}\text { Takers of vitamin } B_{6} \\
{ }^{*} p<.05 \text {, one-tailed. }\end{array}$ & 25.18 & \\
\hline
\end{tabular}

sible for clinical judgments (nursing diagnoses) based on an individual's responses to actual or potential health problems and life processes (Carpenito, 1997). These nursing diagnoses provide the basis for selection of appropriate interventions to achieve positive outcomes. When a pregnant woman is experiencing NVP, acupressure would afford nurses a cost-effective, safe, easily implemented intervention that uses the body's own mechanisms to restore homeostasis and requires no prescription. Acupressure not only may eliminate the physiologic discomforts associated with NVP but also assist in maintaining or increasing productivity as well as reducing disruption in family, emotional, and social functioning. With just one noninvasive intervention, the nurse could achieve many positive outcomes for the pregnant woman experiencing NVP. Further research on other nonpharmacologic modalities is indicated. Nursing has a responsibility to know the effects of nonpharmacologic interventions. 
TABLE 8

Mann-Whitney $U$ Comparison of Independent Samples: Nontakers of Vitamin $B_{6}(n=$ 36) and Takers of Vitamin $B_{6}(n=27)$ Within Group 2 (placebo), Days 5-7

\begin{tabular}{|lccc|}
\hline & $\begin{array}{c}\text { Mean } \\
\text { Rank }\end{array}$ & $\begin{array}{c}\text { p Value } \\
\text { (one- } \\
\text { tailed) }\end{array}$ \\
Average frequency of nausea & & 432.5 & $n s$ \\
Nontakers of vitamin $\mathrm{B}_{6}$ & 30.51 & & \\
Takers of vitamin $\mathrm{B}_{6}$ & 33.98 & & \\
Average severity of nausea & & 450.0 & $n s$ \\
Nontakers of vitamin $\mathrm{B}_{6}$ & 31.00 & & \\
Takers of vitamin $\mathrm{B}_{6}$ & 33.33 & & \\
Average frequency of vomiting & & 403.0 & $n s$ \\
Nontakers of vitamin $\mathrm{B}_{6}$ & 29.69 & & \\
Takers of vitamin $\mathrm{B}_{6}$ & 35.07 & & \\
Average severity of vomiting & & 435.0 & $n s$ \\
Nontakers of vitamin $\mathrm{B}_{6}$ & 30.58 & & \\
Takers of vitamin $\mathrm{B}_{6}$ & 33.89 & & \\
\hline
\end{tabular}

\section{Acknowledgment}

Funding for this study was provided by the Kirkhof School of Nursing, Grand Valley State University, Allendale, MI. Acupressure wrist bands were provided by Sea-Band, U.K., Ltd., Churwalk Hinckley, England.

\section{REFERENCES}

Bayreuther, J., Lewith, G. T., \& Pickering, R. (1994). A double-blind cross-over study to evaluate the effectiveness of acupressure at pericardium $6\left(\mathrm{P}_{6}\right)$ in the treatment of early morning sickness (EMS). Complementary Therapies in Medicine, 2, 70-76.

Beal, M. (1992a). Acupuncture and related treatment modalities. Part 1: Theoretical background. Journal of Nurse Midwifery, 37, 254-259.

Beal, M. (1992b). Acupuncture and related treatment modalities. Part 2: Applications to antepartal and intrapartal care. Journal of Nurse-Midwifery, 37, 260-268.

Belluomini, J., Litt, R. C., Lee, K. A., \& Katz, M. (1994). Acupressure for nausea and vomiting of pregnancy: A randomized blinded study. Obstetrics and Gynecology, $84,245-248$

Brown, S., North, D, Marvel, M. K., \& Fons, R. (1992). Acupressure wristbands to relieve nausea and vomiting in hospice patients: Do they work? American Journal of Hospice of Palliative Care, 7, 26-29.

Campbell, D. T., \& Stanley, J. C. (1963). Experimental and quasi-experimental designs for research. Boston: Houghton Mifflin.
TABLE 9

Mann-Whitney $U$ Comparison of Independent Samples: Nontakers of Vitamin $B_{6}(n=$ 24) and Takers of Vitamin $B_{6}(n=15)$ Within Group 2 (placebo), Days 5-7

\begin{tabular}{|c|c|c|c|}
\hline & $\begin{array}{l}\text { Mean } \\
\text { Rank }\end{array}$ & $\boldsymbol{U}$ & $\begin{array}{c}\text { p Value } \\
\text { (one- } \\
\text { tailed) }\end{array}$ \\
\hline Average frequency of nausea & & 131.5 & $n s$ \\
\hline Nontakers of vitamin $B_{6}$ & 17.98 & & \\
\hline Takers of vitamin $B_{6}$ & 23.23 & & \\
\hline Average severity of nausea & & 154.5 & ns \\
\hline Nontakers of vitamin $B_{6}$ & 18.94 & & \\
\hline Takers of vitamin $B_{6}$ & 21.70 & & \\
\hline Average frequency of vomiting & & 159.5 & ns \\
\hline Nontakers of vitamin $B_{6}$ & 19.15 & & \\
\hline Takers of vitamin $B_{6}$ & 21.37 & & \\
\hline Average severity of vomiting & & 148.5 & ns \\
\hline Nontakers of vitamin $B_{6}$ & 18.46 & & \\
\hline Takers of vitamin $B_{6}$ & 21.10 & & \\
\hline
\end{tabular}

Carpenito, L. J. (1997). Nursing diagnosis: Application to clinical practice. Philadelphia: J. B. Lippincott.

Cohen, J. (1992). A power primer. Psychological Bulletin, $112,155-159$.

De Aloysio, D., \& Penacchioni, P. (1992). Morning sickness control in early pregnancy by Neiguan point acupressure. Obstetrics of Gynecology, 80, 852-854.

Depue, R., Bernstein, L., Ross, R., Judd, H., \& Henderson, B. (1987). Hyper-emesis gravidarum in relation to estradiol levels. Pregnancy outcome and other maternal factors: A seroepidemiologic study. American Journal of Obstetrics and Gynecology, 156, 1137-1140.

Dilorio, C. (1988). Management of nausea and vomiting in pregnancy. Nurse Practitioner, 13(5), 23-28.

Dundee, J. W., Sourial, F.B.R., Ghaly, R. G., \& Bell, P. F. (1988). Acupressure reduces morning sickness. Journal of Royal Society of Medicine, 81, 456-457.

Hsu, C. D., \& Witter, F. R. (1993). Fetal sex and severe hyperemesis gravidarum. International Journal of Obstetrics and Gynecology, 40, 63-64.

Hyde, E. (1989). Acupressure therapy for morning sickness: A controlled study. Journal of Nurse Midwifery, 34, 171-178.

Janfelt-Samsioe, A., Samsioe, G., \& Velinder, G. (1983). Nausea and vomiting in pregnancy-A contribution to its epidemiology. Gynecological and Obstetric Investigations, 16, 221-226.

Klebanoff, M., Koslowe, P., Kaslow, R., \& Rhodes, G. (1985). Epidemiology of vomiting in early pregnancy. Obstetrics and Gynecology, 66, 612-616.

Murphy, P. A. (1998). Alternative therapies for nausea and vomiting of pregnancy. Obstetrics \& Gynecology, 91, 149-155. 
O'Brien, B. (1991). Nausea and vomiting during pregnancy (NVP): A descriptive correlational study. Ann Arbor, MI: University Information Service.

O'Brien, B., \& Naber, S. (1992). Nausea and vomiting during pregnancy: Effect on women's lives. Birth, 19, 138-143.

O'Brien, B., Relyea, M. J., \& Taerum, T. (1996). Efficacy of $P_{6}$ acupressure in the treatment of nausea and vomiting during pregnancy. American Journal of Obstetrics Gynecology, 174, 708-715.

O'Brien, B., \& Zhou, Q. (1995). Variables related to nausea and vomiting during pregnancy. Birth, 22, 93-100.

Ornstein, M., Einarson, A., \& Koren, G. (1995). Bendectin/diclectin for morning sickness: A Canadian follow-up of an American tragedy. Reproductive Toxicology, 9, 1-6.

Pillitteri, A. (1995). Maternal \& child bealth nursing (2nd ed.). Philadelphia: J. B. Lippincott.

Rhodes, V. A., Watson, P. M., \& Johnson, M. H. (1984). Development of reliable and valid measures of nausea and vomiting. Cancer Nursing, 7, 33-41.

Sahakian, V., Rouse, D., Sipes, S., Rose, N., \& Niebyl, J. (1991). Vitamin B6 an effective therapy for nausea and vomiting of pregnancy: A randomized double-blind placebo-controlled study. Obstetrics \& Gynecology, 78, 33-36.

Stainton, M. C., \& Neff, E. J. (1994). The efficacy of Seabands for the control of nausea and vomiting in pregnancy. Health Care for Women International, 16, 563575.

Tierson, F., Olsen, C., \& Hook, E. (1986). Nausea and vomiting of pregnancy and association with pregnancy outcome. American Journal of Obstetric and Gynecology, 155, 1017-1022.

Vellacott, I. D., Cooke, E. J., \& James, C. E. (1988). Nausea and vomiting in early pregnancy. International Journal of Obstetrics and Gynecology, 27, 57-62.

Vutyavanich, T., Wongtrangan, S., \& Ruangsri, R. (1995). Pyridoxine for nausea and vomiting of pregnancy: A randomized double-blind placebo controlled trial. American Journal of Obstetrics and Gynecology, 173, 881-884.

Weinstein, B. B., Wohl, Z., Mitchell, G. J., \& Susendal, G. F. (1944). Oral administration of pyridoxine hydrochloride in the treatment of nausea and vomiting of pregnancy. American Journal of Obstetric and Gynecology, 47, 389-394.

Willis, R. S., Winn, W. W., Morris, A. T., Newsom, A. A., \& Massey, W. E. (1942). Clinical observations in the treatment of nausea and vomiting in pregnancy with vitamin B1 and B6: A preliminary report. American Journal of Obstetrics and Gynecology, 44, 265-271.

Nancy M. Steele is an assistant professor in the Kirkhof School of Nursing, Grand Valley State University, Allendale, MI, and a doctoral student at the University of Michigan, School of Nursing, Ann Arbor.

Joyce French is an associate professor in the Kirkbof School of Nursing, Grand Valley State University, Allendale, MI.

Janet Gatherer-Boyles is a consultant with Boyles, Curtin Associates, Grand Rapids, MI.

Sandra Newman is an instructor for the University of Phoenix, Grand Rapids, MI, and a nurse practitioner at Borgess Women's Health, Portage, MI.

Suzanne Leclaire is a graduate assistant at Grand Valley State University, Allendale, $\mathrm{Mr}$.

Address for correspondence: Nancy M. Steele, RNC, MSN, WHNP, Grand Valley State University, Kirkbof School of Nursing, One Campus Drive, 206 Henry Hall, Allendale, MI 49401-9403. E-mail: steeln@gvsu.edu. 